Periódico do Núcleo de Estudos e Pesquisas sobre Gênero e Direito Centro de Ciências Jurídicas - Universidade Federal da Paraíba Edição Especial - Health, Gender and Human Rights

V. 6 - No 01 - Ano 2018

ISSN | 2179-7137 | http://periodicos.ufpb.br/ojs2/index.php/ged/index

\title{
EL ESTRÉS DE LAS MADRES CON HIJOS/AS CON SÍNDROME DE DOWN: ANÁLISIS DESCRIPTIVO DE UNA MUESTRA ESPAÑOLA
}

Resumen: El artículo describe el estrés de una muestra de madres españolas de niños con síndrome de Down entre 0 y 12 años, a partir de una tesis doctoral que estudió la adaptación familiar a la llegada de un niño con síndrome de Down aplicando para ello el modelo Doble ABCX de McCubbin y Patterson (1983). Los resultados expuestos pueden guiar futuras investigaciones que utilicen muestras mayores y estadísticamente representativas. El número de madres estudiadas es de 161, la mayoría de ellas casadas y en familias biparentales. $\mathrm{Su}$ edad media es 40,7 y proceden en mayor medida de las Comunidades Autónomas de Madrid y Andalucía. El instrumento utilizado ha sido el Índice de Estrés Parental en su versión abreviada de Abidin (1995). Consta de 36 afirmaciones en una escala tipo Likert de 1 a 5. En su versión original el Índice presenta un coeficiente de consistencia
Dra. Dña. Eva M. Rubio Guzmán ${ }^{1}$

Dra. Dña. Rosalía Mota López ${ }^{2}$ interna 0,91. En el presente estudio ésta ha sido de 0,94. La información relativa al resto de variables ha sido recogida mediante un cuestionario original elaborado para la investigación. Los resultados muestran que las madres no presentan un nivel de estrés elevado. Los recursos personales de los que disponen - crianza compartida, elevado nivel formativo e ingresos disponibles -, y el acceso a recursos de apoyo social informal y formal, les permiten afrontar en mejores condiciones la llegada del niño/a con discapacidad. Familiares cercanos, amigos y profesionales constituyen factores protectores de primer orden. Las madres del estudio tienen una valoración bastante positiva de la relación que mantienen con los profesionales desde los primeros momentos del diagnóstico, en relación con la comunicación de la discapacidad de sus hijos y la información y

\footnotetext{
${ }^{1}$ Associate Professor, Faculty of Humanities and Social Sciences, Comillas University

${ }^{2}$ Associate Professor, Faculty of Humanities and Social Sciences, Comillas University
} 
ISSN | 2179-7137 | http://periodicos.ufpb.br/ojs2/index.php/ged/index

orientación inicial que recibieron. Una mayor edad de la madre, una situación económica más fragil, afrontar la crianza sin el otro progenitor, el crecimiento de los hijos, y la no disposición de redes familiares, amicales y profesionales, constituyen en las mujeres estudiadas factores de riesgo para el estrés maternal. Palabras-Clave: estres, síndrome de Down, madres, crianza, familia.

Abstract: The article describes the stress of a sample of Spanish mothers with Down syndrome children between 0 and 12 years, from a doctoral thesis that studied the family adaptation applying the double model ABCX by McCubbin and Patterson (1983). The results presented may guide future research using larger and statistically representative samples. The number of mothers studied is 161 , most of them married and in biparental families. Its average age is 40.7 and live mainly in the Autonomous Communities of Madrid and Andalusia. The instrument used was the Parental Stress Index in its abbreviated version of Abidin (1995). It consists of 36 affirmations on a Likert scale of 1 to 5 . In its original version the Index presents an internal consistency coefficient of 0.91 . In the present study it was 0.94 . The information related to the other variables have been collected through an original questionnaire developed for the research. The results show that mothers do not present a high level of stress. Their personal resources shared parenting, high levels of education and available income - and access to informal and formal social support networks, enable them to cope better with the arrival of children with disabilities. Close relatives, friends, and professionals are relevant protective factors. The mothers of the study have a very positive evaluation of the relationship with professionals since the first moments of the diagnosis, in relation to the communication of the disability of their children and the information and initial orientation that they received. A higher age, a more fragile economic situation, facing the breeding without the other parent, the growth of the children, and the lack of family, amical and professional support, constitute risk factors for stress of the mother studied.

Keywords: Stress, Down syndrom, mothers, upbringing, Family. 


\section{Introducción:}

El presente artículo recoge algunos de los resultados obtenidos en la investigación llevada a cabo en el marco de la elaboración de una tesis doctoral. ${ }^{3}$ Esta investigación trataba de profundizar en la realidad de los padres y las madres que han de afrontar la crianza durante la infancia de un niño o niña con síndrome de Down, siguiendo para ello el modelo Doble ABCX de McCubbin y Patterson (1983). Supone la aplicación por primera vez del modelo Doble ABCX a una muestra de padres y madres de niños/as con síndrome de Down entre 0 y 12 años en España. Este modelo ayuda a ver como ante una misma situación potencialmente estresante por acumulación de demandas (AA), las familias pueden hacer uso de diferentes recursos formales e informales $(\mathrm{BB})$, que sumados al significado que otorga la familia a la citada situación y su sentido de coherencia familiar (CC) puede conllevar una adaptación y buen ajuste familiar. Según este modelo, el factor estresante no tiene por qué conducir necesariamente a una crisis cuando la

3 Rubio Guzmán, E. "La adaptación de las Familias con Hijos/as con Síndrome de Down. Una Aproximación desde el Modelo Doble ABCX. familia utiliza de manera adecuada y efectiva sus recursos y percibe el factor de estrés de una manera más positiva. Por el contrario, la familia desarrollaría una adaptación apropiada que le permitiría hacer frente a la situación estresante y fortalecerse a través de este proceso.

Este trabajo resume los hallazgos desde una perspectiva de género de los datos del citado estudio referidos al estrés parental. Se describe el estrés de una muestra española de madres $\quad(\mathrm{N}=161), \quad$ principales participantes en este tipo de estudios, y las diferencias en su bienestar según algunas variables moduladoras de éste, en concreto, edad de la madre, situación laboral de ésta, nivel de ingresos familiar, tipo de familia, número y edad de los hijos, y apoyo social. El propósito del artículo es fundamentalmente de carácter exploratorio, proponiendo evidencias a partir de una muestra, que puedan ser contrastadas en futuras investigaciones de carácter representativo.

Defendida en la Universidad Pontificia Comillas el 19/1/2016, 


\section{El estrés en las familias con hijos/as \\ con discapacidad intelectual.}

Las familias experimentan a lo largo de su ciclo vital transiciones importantes como nacimientos, matrimonios o defunciones, que se conocen como cambios normativos $\mathrm{y}$ que, aunque suelen ser previsibles, pueden ir acompañadas de tensión y estrés. Aunque el cambio es una parte de la estructura de la vida de las familias, éstas pueden diferir en su capacidad para gestionar dichos cambios, dependiendo de sus recursos internos, fortalezas y capacidades para la resolución de los problemas (McCubbin \& McCubbin, 1993). Sin embargo, hay cambios inesperados e imprevistos, como el nacimiento de un bebé con discapacidad en una familia, que pueden afectarla y requerir una respuesta específica, que en función de su naturaleza, duración e intensidad, puede llevar a las familias a un estado de crisis y a un periodo de desorganización familiar (Rasheed et al., 2001). En estas circunstancias las familias pueden experimentar vivencias de estrés.

El estrés familiar se define
como un estado que surge de un desequilibrio real o percibido entre las demandas que tiene la familia y su capacidad para satisfacerlas. Si una familia no tiene la capacidad de responder a las demandas adicionales o sólo puede hacerlo mediante cambios significativos, uno o más miembros de la familia pueden experimentar estrés (Abery, 2006). Aunque todas las personas sufren una cierta cantidad de estrés y de tensión, si éste es experimentado durante un largo período de tiempo, puede implicar que tanto el individuo como la familia no puedan cubrir con eficacia sus necesidades. Una manifestación más específica de esta respuesta de estrés es lo que Abidin (1992) denomina estrés parental. Este es definido como un proceso en el que los progenitores se sienten desbordados ante las demandas propias del rol de padre o madre, promoviendo la aparición de sentimientos negativos sobre sí mismo como progenitor o sobre el menor

\section{La llegada a la familia de un niño/a con discapacidad intelectual}

La literatura subraya la especial vulnerabilidad al estrés de las familias de los niños y niñas con discapacidad intelectual. Tener un hijo/a con 
discapacidad intelectual en el hogar afecta a la calidad de vida de los miembros de la familia, a los roles de género de los progenitores, a su situación laboral y disponibilidad de recursos financieros, a la disposición y uso del tiempo libre, a la salud y al estrés, e incluso a la aparición de acontecimientos en la vida familiar como el divorcio o el nacimiento de otro niño (Di Giulio, Philipov \& Jaschinski, 2014).

Los estudios sobre discapacidad intelectual han puesto de manifiesto a lo largo del tiempo, que afrontar la parentalidad implica un mayor riesgo de estrés para los progenitores cuando su hijo o hija presenta una discapacidad intelectual (Baker-Ericzén et al., 2005; Dubbs, 2008; Gupta, \& Kaur, 2010; Hastings, 2002; Hassall et al., 2005; Hodapp et al., 2003; Johnston et al., 2003; Keller \& Honig, 2004; Mak \& Ho, 2007; Minnes, 1998; Padeliadu, 1998; Sanders \& Morgan, 1997; Simmerman et al., 2001; Webster et al., 2008). En Estados Unidos, Sanders \& Morgan (1997) encontraron que los padres y madres de niños/as con síndrome de Down preadolescentes experimentaban más estrés y problemas de ajuste que los padres y madres de niños de desarrollo típico. En este sentido, Padeliadu (1998) encontró en Grecia mayores niveles de estrés entre las madres de niños/as con síndrome de Down respecto a las madres de niños sin discapacidad, debido fundamentalmente a la necesidad de invertir más tiempo en la educación y ocio de sus hijos/as. Asimismo, BakerEriczén et al. (2005) al estudiar los niveles de estrés de los padres de niños/as con autismo concluyeron que su nivel de estrés era superior al de los padres de niños/as con desarrollo típico. Por su parte, Gupta \& Kaur (2010) señalaron que el estrés superior sufrido por los padres y madres de niños/as con discapacidad, respecto a los de niños/as sin discapacidad, no sólo era mental sino también físico.

No obstante, diferentes estudios a lo largo del tiempo han venido a contradecir estas visiones, indicando que el tener un hijo/a con discapacidad intelectual no lleva invariablemente a experimentar estrés y dificultades en el funcionamiento parental (Blacher, Baker \& Kaladjian, 2013; Bower \& Hayes, 1998; Byrne \& Cunningham, 1985; Hastings, Allen, Mcdermott, \& Still, 2002; Scorgie, \& Sobsey, 2000). Bower \& Hayes (1998) señalan que las familias de los niños/as con y sin discapacidades de desarrollo tienen características más 
comunes que diferencias. Por su parte, Scott et al. (1997) en un estudio realizado en Canadá con familias de niños/as con síndrome de Down encontraron sólo un 6\% de padres/madres clínicamente deprimidos, frente a un $80 \%$ que no mostraba signos de depresión. Los autores de este estudio señalaron que estos bajos niveles de estrés estaban relacionados con el cambio de visión que se está produciendo en la sociedad sobre la presencia de un niño/a con discapacidad intelectual en la familia y su incidencia en el funcionamiento familiar. Asimismo, Blacher et al. (2013) observaron que el comportamiento de los padres y madres de niños/as entre 3 y 5 años con autismo, parálisis cerebral, síndrome de Down, otras etiologías indiferenciadas y desarrollo típico, presentaba niveles de estrés similares.

El primer momento de vulnerabilidad de las familias con hijos/as con discapacidad intelectual al estrés es cuando reciben la noticia de su diagnóstico. Para los padres y madres el diagnóstico de la discapacidad en un niño/a es habitualmente una experiencia traumática (Green, 2002; Hedov et al, 2002; Keerney \& Griffin, 2001) que les genera múltiples sentimientos de sorpresa, conmoción, angustia, dolor y tristeza (Robledo, 2011; Rodrígues \& Dupas, 2011), de negación, ira, rebelión y rechazo (Silva \& Dessen, 2001; Torres \& Maia, 2009), de miedo o ansiedad (Skotko, 2005) y de culpa, vergüenza y aislamiento (Choi et al., 2011). En el estudio realizado por Canbulat et al., (2014) sobre las experiencias de mujeres turcas al recibir el diagnostico de síndrome de Down de su bebé, todas las participantes indicaron que en ese momento experimentaron ansiedad $\mathrm{y}$ miedo. Sin embargo, es frecuente que cada uno de los progenitores responda de distinta manera ante la discapacidad del hijo/a. En ocasiones, uno de los miembros de la pareja tiene sentimientos protectores intensos hacia el bebé, mientras que el otro manifiesta repulsión o miedo a lo que la discapacidad significa para la familia (Cunniungham, 2011).

Las madres estudiadas por Torres \& Maia (2009) refirieron que el diagnóstico que recibieron sobre el síndrome de Down de su bebé fue tardío, inadecuado e insuficiente en lo informativo. Hedov et al. (2002) estudiaron la percepción que tenían los padres y madres de niños/as nacidos con síndrome de Down entre 1992 y 1993, de la primera información y del apoyo 
obtenido por los departamentos pediátricos suecos. Los resultados revelaron que sólo el $50 \%$ de los progenitores se mostraron satisfechos con la primera información y el apoyo recibido.

Skotko (2005) señala, en relación a la percepción de las madres de su estudio, que los médicos hablaron poco de los aspectos positivos del síndrome de Down y raramente pusieron a su disposición material sobre el asunto, o teléfonos de otros padres o madres con hijos/as con síndrome de Down, para poder intercambiar ideas. Son los progenitores de niños/as con síndrome de Down los que peor valoran, en general, las actitudes de los profesionales, juzgando la comunicación del profesional de la noticia del síndrome de su hijo o hija como rápida y fría, con una transmisión de información inadecuada que, rara vez, aludió a los aspectos positivos del síndrome de Down (Skokto \& Canal, 2004; GAT, 2011).

En este sentido Skotko \& Bedia (2004) se pusieron en contacto con madres que reportaron sentimientos optimistas acerca de tener un niño/a con síndrome de Down en España y encontraron que las madres que habían recibido la información destacando los aspectos positivos del síndrome de Down fueron más positivas y optimistas sobre la crianza de sus hijos/as. Esto hace pensar que los progenitores a los que se da la noticia del problema de su hijo o hija de forma amable y comprensiva, ofreciéndoles información precisa y apoyo, aceptan el diagnóstico con más facilidad (Cunniungham, 2011).

\section{Dificultades en la crianza de un hijo con Discapacidad Intelectual}

\section{La unidad familiar}

Tener un hijo/a con discapacidad intelectual influye en la relación de la pareja, pudiendo actuar bien como desencadenante de una ruptura, sobre todo cuando ya existían conflictos previos, o bien como un factor de unión y fortalecimiento de la relación (FEAPS, 2008). En cualquier caso, la familia tiene una ruptura inicial en su equilibrio sistémico que se irá restableciendo en la medida en la que se produzca su adaptación a la nueva situación (Acero Rodríguez, 2013).

Durante décadas, se consideró que tener un hijo/a con discapacidad intelectual generaba más conflictos en la pareja 
(Kersh et al., 2006) que disminuían la satisfacción con la relación (Friedich \& Friedrich, 1981) y que desembocaban en muchos casos en la ruptura y el divorcio (Cooke et al., 1986; Hogan et al., 2012). Gath (1978) comparando las familias de niños/as con síndrome de Down con las familias de niños/as con desarrollo típico, encontró que en las primeras había una mayor incidencia de divorcio y conflictos maritales durante los años de la infancia.

Algunos estudios indican que los menores con discapacidad tienen mayor riesgo de crecer en un hogar monoparental (Loft, 2011, 2013; Manjarrés, 2012). Según indica Manjarres (2012) la crianza de personas con discapacidad se identifica con la ausencia de figuras parentales, en mayor proporción esa ausencia es del padre, pero en algunos casos es de la madre o de ambos.

\section{El reparto de roles de género entre el} padre y la madre.

Aunque la discapacidad intelectual del hijo/a afecta a los padres y las madres, su impacto es desigual en cada uno de ellos. Muy probablemente esto se deba a la influencia, muchas veces determinante, de los estereotipos de género dentro de la familia.

Las madres juegan un papel clave en la adaptación de la familias, asumen mayor carga y se configuran como cuidadoras primarias (FEAPS, 2008), renunciando a su actividad laboral y encargándose a tiempo completo del cuidado y crianza de su hijo o hija, sujetándose así a las demandas sociales que pesan sobre la maternidad (Núñez, 2010). Habitualmente, es la madre quien se ocupa de todo el seguimiento médicorehabilitador, educativo y social del menor (Mercado \& García, 2010). Por su parte los padres suelen estar más ausentes en el cuidado directo de su hijo/a, implicándose más en tareas indirectas de gestión y manutención de la familia (Cunniungham, 2011).

Esta distribución desigual de papeles en ocasiones es motivada y potenciada, según Núñez (2010), por los profesionales que delegan más en las madres que en los padres el seguimiento y tratamiento del hijo/a con discapacidad intelectual.

Por lo tanto, la presencia de un niño/a con discapacidad podría alentar a la familia a adoptar roles tradicionales de género, no sólo porque suelen requerir una mayor cantidad de atención, sino 


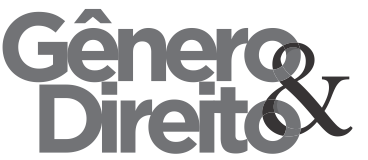

Periódico do Núcleo de Estudos e Pesquisas sobre Gênero e Direito

Centro de Ciências Jurídicas - Universidade Federal da Paraíba

Edição Especial - Health, Gender and Human Rights

V. 6 - $\mathrm{N}^{\circ} 01$ - Ano 2018

ISSN | 2179-7137 | http://periodicos.ufpb.br/ojs2/index.php/ged/index

también porque los roles de cuidador no

son fácil ni convenientemente transferidos (Di Giulio et al., 2014).

En las conclusiones del II Congreso Nacional de Familias con Personas con Discapacidad Intelectual (2003) las madres reclamaban una mayor implicación de los padres indicando que no les bastaba con que éstos se limitaran a colaborar, sino que querían que se comprometieran a compartir, en pie de igualdad, las tareas de cuidado de sus hijos/as. Florez (2000, p.121) destaca la necesidad de que los padres asuman la misma responsabilidad que las madres en la crianza y educación de un hijo/a, pero más aún cuando éste presenta una discapacidad, por el necesario trabajo, esfuerzo y empeño. Así indica que "cuanta mejor voluntad se pone, mayor es el cansancio y el riesgo de agotamiento. Son más las tareas que hay que hacer. Son más especiales los métodos educativos que hay que aplicar, que no se aprenden por simple intuición sino que exigen un cierto estudio $y$ observación".

A pesar de esta distribución diferencial de papeles en función del género, este reparto de roles está cambiando, percibiéndose una presencia cada vez mayor de los varones en el cuidado y atención directa de los hijos e hijas con discapacidad intelectual (FEAPS, 2008; Cunniungham, 2011).

\section{Vida laboral de las madres}

Por otro lado, hemos de resaltar la incidencia que tiene la discapacidad intelectual de un hijo o una hija en la vida laboral de sus padres y madres (fuente primordial de ingresos familiares), por las consecuencias económicas que plantea. En ocasiones las madres (muy frecuentemente por el tipo de empleo que ocupan y/o por las expectativas del rol de género) ven restringidas sus oportunidades de empleo (Cunningham, 1996) y se ven obligadas a renunciar a su puesto de trabajo (y por lo tanto a la remuneración percibida), dadas las altas exigencias de cuidado del menor y las dificultades reales para la conciliación de la vida laboral y la vida familiar, con el consiguiente incremento del impacto económico negativo en las familias (Chichevska Jovanova \& Dimitrova Radojichikj, 2013; FEAPS, 2008, Núñez, 2010; Phelps et al. 2012; Rogers \& Hogan 2003). Las madres también suelen encontrar limitaciones en relación al tipo de empleo al que pueden acceder o al aprovechamiento de las 
oportunidades laborales de promoción, desarrollo y crecimiento profesional que pudieran surgirles, debido a las necesidades de supervisión de su hijo/a (Cunniungham, 2011). Según el estudio europeo realizado por Di Giulio et al. (2014) los varones con un hijo/a con discapacidad también presentan una menor participación en el mercado de trabajo.

\section{El estrés de las madres de un niño/a con discapacidad.}

Las investigaciones en su aproximación a la situación de las familias que tienen en su seno un hijo/a con discapacidad intelectual, se han centrado tradicionalmente en la experiencia de las madres, como protagonistas principales del cuidado $\mathrm{y}$ atención de los menores (Hodapp, 2007; Pozo et al., 2006). Tomanik et al. (2004) señalan que dos tercios de las madres de los niños/as diagnosticados con una discapacidad del desarrollo pueden experimentar niveles elevados de estrés. Este acercamiento a las madres se ha realizado fundamentalmente desde una perspectiva comparativa, en primer término, con las madres de niños/as sin discapacidad y, en segundo término, con los padres de niños/as con discapacidad intelectual. Asimismo, Azar \& Kurdahi (2006) señalaban que un alto porcentaje de las madres de niños/as con discapacidad intelectual en el Líbano tenía síntomas depresivos.

Al comparar las experiencias de maternidad de hijos/as con y sin discapacidad intelectual, diferentes estudios han demostrado, que las madres de niños/as con discapacidad intelectual presentan mayores tasas de depresión que las madres de niños/as con desarrollo típico (Shapiro, et al., 1998; Hedov, et al., 2000; Sayed et al., 2006). Hedov, et al. (2000) al investigar en Suecia la existencia de posibles diferencias en la salud autopercibida en las madres de niños/as con síndrome de Down y madres de los niños/as sin discapacidad, encontraron que las primeras experimentaban más a menudo fatiga y se sentían más nerviosas y depresivas la mayoría del tiempo, por lo que concluyeron que la presencia de un niño/a con discapacidad intelectual tiene un efecto negativo en las madres, dando lugar a déficits en su salud.

Lalvani (2011), en un estudio realizado para explorar la experiencia de las madres tras el nacimiento de su hijo con síndrome de Down, destacó 
numerosas formas en las que las familias fueron colocadas en el discurso hegemónico como "el otro". Las madres objeto de estudio indicaron que además de ser beneficiarias de la simpatía de personas en su entorno lo eran de la admiración, al recibir mensajes que las calificaban de "valientes", "con coraje", "bendecidas" o "elegidas por Dios" para tener un bebé "especial". Estos mensajes, aunque bien intencionados, transmitían que sus hijos e hijas no eran "normales" o "perfectos" y por lo tanto no eran deseables. Estas visiones socioculturales, que construyen, apoyan y sostienen las emociones de tristeza, pena $\mathrm{y}$ angustia, permanecen en gran medida sin examinar en los estudios que analizan los niveles de estrés de los progenitores de niños y niñas con discapacidad intelectual (Lalvani, 2008, 2011).

No obstante, no todos los estudios vienen a confirmar estas diferencias entre las madres de grupos familiares diferenciados por la presencia o no de un hijo/a con discapacidad intelectual. En este sentido, Bower et al. (1998) analizaron y compararon, en Australia y Nueva Zelanda, la percepción de resistencia familiar que tenían las madres de niños/as con síndrome de Down entre 5 y 15 años y las madres de niños/as sin discapacidad, no encontrando diferencias significativas en la dinámica familiar entre ambos grupos. Asimismo, el estudio de Emerson (2003) reveló que aunque muchas madres de los niños/as con discapacidad intelectual presentaban un estrés adicional en su vida, sólo una minoría de ellas presentaba resultados psicológicos, sociales o familiares adversos.

Por otro lado, las investigaciones ponen de relieve que las madres y los padres experimentan un impacto diferente y tienen necesidades diferentes para afrontar la discapacidad de su hijo/a (Neely-Barnes \& Día, 2008). La mayoría de los estudios indican que las madres tienen mayores tasas de depresión y experimentan más problemas de ajuste psicológico que los padres (Aldosari \& Pupaff, 2014; Azar \& Kundahi, 2006; Dabrowska \& Pisula, 2010; Glidden \& Floyd, 1997; Gray, 2003; Hastings et al., 2005; Hasting et al., 2006; Negrin \& Cristante, 1996; Oelofsen \& Richardson, 2006; Shin, et al., 2006; Singer, 2006). Gray (2003) en su estudio sobre familias con hijos/as con autismo encontró que la discapacidad de su hijo/a afectaba a los padres y madres de forma distinta, por los distintos roles 
de género, que implicaban una mayor presencia de las madres en la crianza. También halló diferencias de género en las estrategias de afrontamiento, dado que las madres tendía a expresar sus sentimos y los padres a suprimirlos o evitarlos, trabajando fuera de casa hasta tarde.

En este mismo sentido, tanto Shin et al. (2006) al estudiar el estrés percibido por las familias de niños/as con discapacidad intelectual en Vietnam, como Aldosari \& Pufpaff (2014) al hacerlo en Arabia Saudita, hallaron niveles de estrés más altos en las madres. Aldosari \& Pufpaff (2014) se apoyan, para explicar estos resultados, en aspectos culturales y de género, dado que en la cultura saudí las mujeres son las principales cuidadoras. Por su parte, Dabrowska \& Pisula (2010) al investigar las familias de niños/as con autismo y con síndrome de Down, encontraron puntuaciones más altas en estrés en las madres que en los padres.

Asimismo, al combinar tanto la presencia de la discapacidad como la perspectiva de género, los resultados del estudio de Olsson \& Hwang (2001) sobre las familias con niños con autismo y/o discapacidad intelectual (entre 0 y 16 años), indicaban que las madres de los niños/as con discapacidad presentaban puntuaciones de depresión más altas que las madres de niños/as sin discapacidad y que los padres de niños/as con discapacidad, al igual que sucedía en el estudio de Basa (2010) en Argentina sobre padres y madres de niños/as con autismo. En este mismo sentido, Oelofsen \& Richardson (2006) al estudiar en Suecia a las familias con niños en edad preescolar con y sin discapacidad del desarrollo, encontraron que el $84 \%$ de las madres de niños/as con discapacidad del desarrollo presentaba niveles de estrés de rango clínico frente al $67 \%$ de los padres; asimismo, las madres reportaron mayores problemas de salud. Por su parte Pereira-Silva (2006), en una investigación llevada a cabo para analizar el funcionamiento de las familias con hijos/as con síndrome de Down en comparación con las familias de niños/as con desarrollo típico, concluyeron que buena parte de las primeras presentaban una puntuación más alta en estrés que las madres de los niños/as con desarrollo típico. Sin embargo, en el caso de los padres el resultado fue inverso, es decir, los padres de niños/as de desarrollo típico percibieron más estrés en sus relaciones con sus hijos/as que los padres de 
niños/as con síndrome de Down. No obstante, al comparar a las madres con los padres de niño/as con síndrome de Down, estos últimos presentaron puntuaciones más bajas en estrés que las madres.

Esta tendencia de mayor presencia de estrés en las madres que en los padres es contravenida por algunos estudios como el de Shonkoff et al. (1992) que encontró que los padres reportaban continuamente niveles de estrés más altos que las madres en su trato con los niños/as. En este sentido, muchos padres de esta investigación indicaron que se sentían menos satisfechos en su rol como padres porque su hijo/a tenía síndrome de Down. Asimismo, diversos estudios concluyen que son pocos los datos que existen sobre la figura del padre en relación al funcionamiento de la familia con hijos/as con discapacidad intelectual, al centrarse la mayor parte de los estudios en el cuidador principal (Ortega et al., 2007; Pereira-Silva \& Dessen, 2003).

No obstante, algunos autores señalan que no hay diferencias en la percepción del estrés y el ajuste familiar entre padres y madres de niños/as con discapacidad intelectual (Dyson, 1991, 1997). El estudio que llevaron a cabo
Azar \& Badr (2010) en el Líbano vino a demostrar que ambos, padres y madres de niños/as con discapacidad intelectual, experimentaron de manera similar estrés similares, apoyo social informal y conductas de afrontamiento.

\section{Metodología}

Participantes

La población de estudio son las madres de niños/as con síndrome de Down entre 0 y 12 años de todo el territorio español. La muestra está compuesta por 203 personas, 161 madres y 42 padres. En términos porcentuales las madres representan el 79,3\% de la muestra y los padres el restante $20,7 \%$.

La edad media de las madres descritas es de 40,7 años, con una desviación típica de 5,9 años. Los grupos de edad más numerosos son el de 36-40 años, con un $33,5 \%$ de las madres, y el de 41-45 años, en el que se localizan el 26,7 de ellas. En las edades más extremas, 26-30 años y 51-55, hay un $3,1 \%$ y un $5,6 \%$ respectivamente. La edad mediana de las madres es de 40 años.

Dado el pequeño tamaño de padres encuestados, en la presentación de los resultados se ha optado por no 
seguir una estrategia comparativa entre madres y padres, ya que cualquier desagregación de la población de padres resulta en clasificaciones de población poco significativas.

En cuanto a la procedencia de la muestra se observa una mayor concentración de madres en las Comunidades Autónomas de Madrid y de Andalucía, existiendo una enorme dispersión en el resto del estado español. Un tercio de la muestra vive en la Comunidad de Madrid (33,5\%), en Andalucía lo hace el 29,2\%, el 9,9\% vive en Extremadura, el $6,8 \%$ vive en Cataluña, el $5,0 \%$ en la Comunidad Valenciana, y las madres que viven en otras Comunidades autónomas no superan el 3\%. A destacar que no se han podido conseguir respuestas de familias que vivan en Castilla y León, Navarra, Islas Baleares o en Ceuta y Melilla.

\section{Variables e Instrumento.}

El "Cuestionario de Adaptación familiar con hijos con síndrome de Down entre 0 y 12 años" que se utilizó en la investigación doctoral está formado por un cuestionario sociodemográfico (de elaboración propia) y 5 escalas, todas ellas cumplimentadas directamente por el propio encuestado.

En el artículo se presentan los datos descriptivos del Índice de Estrés Parental (PSI-SF) en su versión abreviada de Abidin (1995). El resto de escalas utilizadas fueron: Escala de Evaluación de la Adaptabilidad y de la Cohesión Familiar (FACES II- VE) en su versión reducida en español (Martínez Pampliega et al., 2006); Índice de Apoyo Social (SSI) (Cooke et al., 1988); Escala de Evaluación Personal del Funcionamiento Familiar en situaciones de crisis (F-COPES) (Ruano Piera \& Serra Desfilis, 2000); Escala de Sentido de Coherencia Familiar (FSOC) (Antonovsky, 1987), y Escala de Satisfacción Familiar (FSfS)(Sanz, 2008).

El Índice de Estrés parental fue creado por Richard Abidin (1983) para evaluar el nivel de estrés de los progenitores derivado del ejercicio de su rol de padres (Díaz-Herrero et al., 2010). En su forma original presentaba 120 items, recogidos en dos subescalas. Dadas las dificultades para su administración, Abidin (1995) ideó una versión reducida de 36 ítems (PSI- SF) que evalúa el estrés que pueden experimentar los padres y madres en el 
ejercicio de la paternidad/maternidad, provocado por las características de los padres y madres, por ciertos rasgos conductuales del niño/a y/o por variables situacionales que se relacionan directamente con el rol parental (DíazHerrero et al., 2010, 2011). Esta versión es la que se ha utilizado en el presente estudio.

El PSI-SF está compuesta por 36 afirmaciones ante las que los padres y madres han de indicar su grado de acuerdo en una escala tipo Likert de 5 que va de 1 "muy en desacuerdo" a 5 "muy de acuerdo". El rango de la escala es por tanto de 34 puntos, que indica el nivel de estrés más bajo, hasta 180 puntos, siendo esta puntuación la de mayor estrés. El Índice consta de las siguientes subescalas, para cada una de las cuales la puntuación mínima es 12 puntos y la máxima 60:

1. La subescala de Malestar Paterno (ítems del 1 al 12) determina el malestar que experimentan los progenitores en su desempeño del papel de padres y madres, provocado por factores personales que están directamente relacionados con el ejercicio de las funciones derivadas de este papel (sentido de competencia, tensiones asociadas con las restricciones impuestas a otras funciones que desarrollamos en la vida, conflictos con el otro progenitor, falta de apoyo social, depresión, etc.).

2. La subescala de Interacción Disfuncional Padres-Hijo (ítems del 13 al 24) se centra en la percepción que los padres y madres tienen del grado en que su hijo/a satisface o no las expectativas que tenían sobre él o ella y del grado de reforzamiento que su hijo/a les proporciona como padres. En esta subescala el ítem 22 es inverso.

3. La tercera subescala es la de Niño Difícil (ítems del 25 al 36), y ofrece una valoración de cómo perciben los progenitores la facilidad o dificultad para controlar a sus hijos/as en función de los rasgos conductuales que presentan. En esta subescala el ítem 32 es inverso.

Este instrumento fue ligeramente modificado al ser introducido en el cuestionario utilizado 


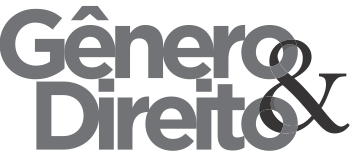

en esta investigación. Dicha modificación consistió en hacer referencia explícita al hijo/a con síndrome de Down en todas las afirmaciones del PSI-SF en donde se incluía la palabra "hijo". Esto ha sido necesario al tener como intención valorar el efecto del hijo/a con síndrome de Down en el padre o madre encuestado, al
Periódico do Núcleo de Estudos e Pesquisas sobre Gênero e Direito Centro de Ciências Jurídicas - Universidade Federal da Paraíba Edição Especial - Health, Gender and Human Rights

V. 6 - $\mathrm{N}^{\mathrm{o}} 01$ - Ano 2018

ISSN | 2179-7137 | http://periodicos.ufpb.br/ojs2/index.php/ged/index

margen de la existencia de otros hijos/as en la familia. De esta forma los ítems que no han sido modificados son: $1,3,6,7$, $9,10,11,12$ у 22 .

El Índice de Estrés Parental en la versión reducida de Abidín (1995) presenta un coeficiente de consistencia interna 0,91. En el presente estudio la consistencia interna ha sido de 0,94 .

Cuadro 6: Ítems y Subescalas del Índice de Estrés Parental en su versión reducida (PSI-SF)

\begin{tabular}{|c|c|}
\hline ITEM & SUBESCALA \\
\hline 1. A menudo tengo la sensación de que no puedo controlar bien las situaciones. & \multirow{12}{*}{$\begin{array}{l}\text { MALESTAR } \\
\text { PATERNO }\end{array}$} \\
\hline $\begin{array}{l}\text { 2. Siento que dejo más cosas de mi vida de lo que nunca imaginé para satisfacer las } \\
\text { necesidades de mi(s) hijo (s) }\end{array}$ & \\
\hline 3. Me siento atrapado por mis responsabilidades como padre/madre. & \\
\hline 4. Desde que he tenido este hijo no he sido capaz de hacer cosas nuevas y diferentes. & \\
\hline $\begin{array}{l}\text { 5. Desde que he tenido este hijo/a, siento que casi nunca soy capaz de hacer las cosas que } \\
\text { me gustan. }\end{array}$ & \\
\hline 6. No me siento contento con la ropa que me compré la última vez. & \\
\hline 7. Hay muchas cosas de mi vida que me molestan & \\
\hline $\begin{array}{l}\text { 8. Tener a mi hijo me ha causado más problemas de los que esperaba en mis relaciones } \\
\text { con mi pareja. }\end{array}$ & \\
\hline 9. Me siento solo/a y sin amigos. & \\
\hline 10. Generalmente, cuando voy a una fiesta no espero divertirme & \\
\hline 11. No estoy tan interesado por la gente como antes.. & \\
\hline 12. No disfruto con las cosas como antes. & \\
\hline 13. Mi hijo/a casi nunca hace cosas que me hagan sentir bien. & \multirow{9}{*}{$\begin{array}{l}\text { INTERACCIÓN } \\
\text { DISFUNCIONAL } \\
\text { PADRES-HIJOS }\end{array}$} \\
\hline 14. Casi siempre siento que no le gusto a mi hijo ni quiere estar cerca de mi. & \\
\hline 15. Mi hijo me sonríe mucho menos de lo que yo esperaba. & \\
\hline $\begin{array}{l}\text { 16. Cuando le hago cosas a mi hijo, tengo la sensación de que mis esfuerzos no son } \\
\text { apreciados. }\end{array}$ & \\
\hline 17. Cuando juega, mi hijo/a no se ríe con frecuencia. & \\
\hline 18. Me parece que mi hijo/a no aprende tan rápido como la mayoría de los niños. & \\
\hline 19. Me parece que mi hijo/a no sonríe tanto como los otros niños. & \\
\hline 20. Mi hijo/a no es capaz de hacer tantas cosas como yo esperaba. & \\
\hline 21. Mi hijo/a tarda mucho y le resulta muy difícil acostumbrarse a las cosas nuevas. & \\
\hline
\end{tabular}


Periódico do Núcleo de Estudos e Pesquisas sobre Gênero e Direito Centro de Ciências Jurídicas - Universidade Federal da Paraíba Edição Especial - Health, Gender and Human Rights

V. 6 - No 01 - Ano 2018

ISSN | 2179-7137 | http://periodicos.ufpb.br/ojs2/index.php/ged/index

22. Siento que soy :

1. No muy bueno como madre/padre.

2. Una persona que tiene algunos problemas para ser madre/padre

3. Un/a madre/padre normal

4. Un/a madre/padre mejor que el promedio

5. Muy buen/a madre/padre

INTERACCIÓN

DISFUNCIONAL

PADRES-HIJOS

23. Esperaba tener más sentimientos de proximidad y calor con mi hijo/a de los que tengo, y eso me molesta

24. Algunas veces mi hijo/a hace cosas que me molestan sólo por el mero hecho de hacerlas.

25. Mi hijo/a parece llorar y quejarse más a menudo que la mayoría de los niños.

26. Mi hijo/a generalmente se despierta de mal humor.

27. Siento que mi hijo/a es muy caprichoso y se enoja con facilidad.

28. Mi hijo/a hace algunas cosas que me molestan mucho.

29. Mi hijo/a reacciona muy fuertemente cuando sucede algo que no le gusta.

30. Mi hijo/a se molesta fácilmente por las cosas más insignificantes.

31. El horario de comer y dormir de mi hijo/a fue mucho más difícil de establecer de lo que yo esperaba.

32. He observado que lograr que mi hijo/a haga o deje de hacer algo es:

1. Mucho más difícil de lo que imaginaba.

NIÑO DIFÍCIL

2. Algo más difícil de lo que esperaba.

3. Como esperaba

4. Algo menos difícil de lo que esperaba

5. Mucho más fácil de lo que esperaba.

33. Piense concienzudamente y cuente el número de cosas que le molesta que haga su hijo/a. Por ejemplo, pierde el tiempo, no quiere escuchar, es demasiado activo, llora, interrumpe, pelea, lloriquea, etc. Por favor, marque el numero que indica el conjunto de cosas que haya contado.

$$
+10,8-9,6-7,4-5,1-3
$$

Por favor indique alguna

34. Algunas de las cosas que hace mi hijo/a me fastidian mucho.

35. Mi hijo/a se ha convertido en un problema mayor de lo que yo esperaba .

36. Mi hijo me exige más de lo que exigen la mayoría de los niños.

Fuente: Elaboración Propia.

Por su parte, la información relativa a la disposición de apoyos sociales ha sido extraída de la aplicación del Índice de Apoyo Social, creado por por Cooke, Rossmann, McCubbin \& Patterson (1988). El apoyo social es 
ISSN | 2179-7137 | http://periodicos.ufpb.br/ojs2/index.php/ged/index

De este instrumento se han utilizado en el presente trabajo sólo las preguntas dicotómicas para determinar los recursos de apoyo que tiene la persona. Las fuentes de apoyo por las que se pregunta son: pareja, hijos, otros familiares, amigos íntimos, compañeros de trabajo, vecinos, comunidad religiosa, profesionales, grupos de apoyo, creencias religiosas y medios de comunicación.

Por su parte, la información relativa a las características sociodemográficas de las madres, el tipo de familia, número y edad de los hijos, y las circunstancias del diagnóstico, fueron recogidas a través del cuestionario que se elaboró específicamente para la investigación.

\section{Procedimiento.}

Dadas las dificultades para establecer un marco muestral definido, y por lo tanto no poder construir una muestra estadísticamente representativa (Cea D’Ancona, 1999), se optó por un muestreo no probabilístico estratégico, en el que la selección de las unidades

\footnotetext{
${ }^{4}$ La relación de entidades vinculadas a Down España puede consultarse en la siguiente dirección: http://www.sindromedown.net/conocenos/nuestras -entidades/
}

muestrales responde a los objetivos de la investigación señala Corbetta (2007, p289), este tipo de muestreo, aunque no presenta las posibilidades de generalización de los muestreos probabilísticos, es apropiado en muestras pequeñas "para garantizar que la información recopilada esté completa”.

Para localizar a las familias que potencialmente pudieran participar en este estudio se utilizaron utilizado dos vías principales: las entidades de síndrome de Down, localizadas principalmente a través de Down España $^{4}$, y los colegios de educación especial que existen en todo el Estado español. Se les pidió a las entidades que hicieran llegar el cuestionario a todas las familias socias que cubrieran el criterio de edad de los menores con síndrome de Down establecido en este estudio. No fue posible contactar con todas las entidades de síndrome de Down y otras colaboradoras. De las 87 iniciales se logró contactar con 76, de las cuales 46 confirmaron su participación.

Por otro lado se obtuvo un listado con los centros de educación especial $^{5}$ de la mayor parte de las

\footnotetext{
5 Los centros de Educación Especial pueden encontrarse en la siguiente dirección: http://sid.usal.es/preguntas-
} 


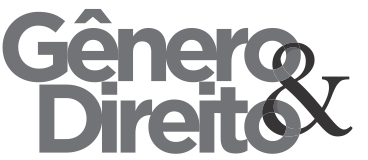

Comunidades Autónomas, tanto

públicos como concertados, con los que se contactó igualmente para presentar el proyecto y pedir su colaboración. De los 231 centros listados, 116 ofrecieron su colaboración para hacerles llegar el estudio a las familias de niños/as con síndrome de Down de sus centros que cumplieran el requisito de edad de los menores. La mayor parte de los centros educativos que no han colaborado ha sido por no contar en sus aulas niños/as con síndrome de Down entre 0 y 12 años.

El inicio de los contactos comenzó en Febrero de 2014 y finalizó el 30 de Junio, con la recogida de los cuestionarios cumplimentados.

\section{Resultados.}

¿Quiénes son las madres de niños con síndrome de Down?

En relación con el estado civil de las madres participantes, el 76,4\% están casadas y el 5,6\% tienen una pareja de hecho. Sólo el 9,9\% son solteras, el $2,5 \%$ separadas, el $5 \%$ divorciadas y el $0,6 \%$ viudas. eriódico do Núcleo de Estudos e Pesquisas sobre Gênero e Direito Centro de Ciências Jurídicas - Universidade Federal da Paraíba Edição Especial - Health, Gender and Human Rights V. 6 - No 01 - Ano 2018 SSN | 2179-7137 | http://periodicos.ufpb.br/ojs2/index.php/ged/index

Asimismo, las madres estudiadas viven en su mayoría en pareja (90,7\%), viviendo el 9,3\% sin pareja. En este sentido el $80,7 \%$ señalan formar parte de una familia biparental, el 14,3\% una familia monoparental y el 5\% forman otro tipo de familia. La mitad de ellas tienen dos hijos $(48,4 \%)$, un $23,6 \%$ de las madres sólo tienen al hijo/a con síndrome de Down, y el 18\% tienen tres 3 hijos. Así, 9 de cada 10 tienen entre uno y tres hijos. Casi una cuarta parte de las mujeres son madres de hijo único. Dos tercios de ellas tienen hijos menores de 7 años, y un $36 \%$ tiene hijos de 0 a 3 años. El nivel de estudios de las madres encuestadas es predominantemente alto, ya que el $55,9 \%$ tienen estudios universitarios medios o superiores. Sólo el 1,9\% no presentan estudios, el 13,0\% tienen estudios primarios y el 28,6\% tienen estudios secundarios.

En relación a la situación laboral de las madres, más de la mitad se encuentran en activo (55,9\%), estando el $34,8 \%$ en situación de desempleo, el $3,1 \%$ en excedencia y el $6,2 \%$ en otra situación. Nos encontramos con una variedad importante de ocupaciones, 
destacando que el $25,9 \%$ señala como ocupación "ama de casa" o cuidadora de sus hijos.

En relación al nivel de ingresos familiares, el 12,4\% ingresa menos de 600 euros mensuales, y para el 21,7\% dichos ingresos ascienden hasta 1200 euros. El 13,7\% tiene ingresos entre 1200 y 1800 euros, que son superados por el $52,2 \%$ restante de la muestra. El valor más frecuente es el que se encuentra entre los 2400 y 3600 euros mensuales $(24,2 \%)$.

Casi la mitad de las madres que crían a sus hijos solas están situadas en el nivel de ingresos bajos (hasta 1200 euros mensuales) $48,0 \%$, mientras que en el caso de las madres que viven en familias biparentales hay un $43,5 \%$ que tienen ingresos familiares altos (más de 2400 euros mensuales)

Las madres de la investigación cuentan con familiares próximos y amigos íntimos que pueden brindarles apoyo en un alto porcentaje, 91,3\% y $84,5 \%$ respectivamente. 6 de cada 10 dicen también disponer de compañeros de trabajo cercanos. Casi la totalidad de ellas tienen contacto con profesionales, 96,9\%. Grupos formales o informales en el vecindario, parroquias o comunidades religiosas, y grupos especiales de apoyo, tienen menos presencia: $75,8 \%$ de las madres dicen no estar implicados en esos grupos en su vecindario, $68,3 \%$ no pertenecen a comunidades religiosas $\mathrm{y}$ $41,6 \%$ tampoco lo hace a ningún grupo especial diseñado específicamente para ayudarle.

\section{El diagnóstico de la llegada de un niño con síndrome de Down a la familia.}

Una amplia mayoría de las madres $(84,5 \%)$ recibió el diagnóstico de síndrome de Down de su hijo o hija tras su nacimiento. Sólo en el 15,5\% de los casos las pruebas prenatales les habían informado de este diagnóstico.

El profesional que informó a las madres del diagnóstico fue principalmente el médico $(86,8 \%)$. Al $3,1 \%$ le informó la enfermera, al 3,1\% la matrona, al 1,2\% el trabajador social y al $5,7 \%$ otra persona (mayoritariamente su marido u otro familiar).

El profesional que más impactó o marcó en los primeros momentos fue el médico para el $75,4 \%$ de las madres, seguido de la enfermera para el 6,9\%, la matrona para el 6\%, el psicólogo para el $3,1 \%$ y el trabajador social para el $1,3 \%$ de las madres.

Más de la mitad de las madres considera positiva la atención que 
recibió por parte del profesional que le informó del diagnóstico, valorándola el $57,9 \%$ como adecuada o totalmente adecuada, así como las orientaciones recibidas que el $57,2 \%$ las califica de adecuadas o totalmente adecuadas. El $9,2 \%$ de las madres consideran la atención de manera indiferente y el $15,1 \%$ realizan la misma valoración de las orientaciones profesionales recibidas. Para el 32,9\% de las madres dicha atención fue inadecuada o totalmente inadecuada y el 27,6\%valoran de la misma manera la orientación profesional.

Tras el diagnóstico, el 85,6\% de las madres acudió a una entidad (asociación, fundación, etc.) relacionada con el síndrome de Down. En este caso la valoración que hacen de la acogida recibida es mayoritariamente positiva. Así, el 98,5 de las madres que acudieron a la entidad valoran la acogida como adecuada o totalmente adecuada; también lo hacen así con la información que les ofrecieron, $(97,8 \%)$, y el apoyo recibido, considerado por el $94,2 \%$ como adecuado o totalmente adecuado.

El estrés de las madres de un niño con síndrome de Down.
El índice de estrés parental en su versión reducida (PSI-SF) mide el estrés de los padres por medio de tres subescalas: Malestar Paterno/Materno, Interacción Disfuncional padres-hijo y Niño Difícil. La media obtenida por las madres estudiadas en la escala de estrés es de 68 y la desviación típica 21,2 en un rango entre 36 y 180 (habiéndose obtenido un rango máximo en los resultados de la escala de 151).

En relación a la subescala Malestar Materno, la media obtenida por las madres estudiadas es de 25,2 , y la desviación típica es 9,34, en un rango entre 12 y 60 (habiéndose obtenido un rango máximo de 57 en los resultados de la subescala).

La puntuación media obtenida en la subescala de Interacción Disfuncional Padres-Hijos es de 20,93 y la desviación típica es 7,3, en un rango entre 12 y 60 (habiéndose obtenido un rango máximo en los resultados de esta subescala de 53).Por ultimo

Por último, en relación a la subescala de Niño difícil, la media obtenida por las madres de este estudio es 21,86 y la desviación típica es 7,99, en un rango entre 12 y 60 (habiendo obtenido un rango máximo en estos resultados de 50). 
Periódico do Núcleo de Estudos e Pesquisas sobre Gênero e Direito Centro de Ciências Jurídicas - Universidade Federal da Paraíba Edição Especial - Health, Gender and Human Rights V. 6 - $\mathrm{N}^{\mathrm{o}} 01$ - Ano 2018

ISSN | 2179-7137 | http://periodicos.ufpb.br/ojs2/index.php/ged/index

Diferencias en el estrés de las madres.

En este apartado se compara el nivel de estrés de las madres de niños con síndrome de Down según su perfil sociodemográfico, composición familiar, y disposición de apoyo social. Se consideran las variables de edad, situación laboral y nivel de ingresos de las madres, tipo de familia, número y edad de los hijos, y si tienen $o$ no las fuentes de apoyo identificadas en la metodología. Conviene valorar con prudencia los resultados, dado el pequeño tamaño de algunos de estos grupos de madres, así como la elevada dispersión que presentan las puntuaciones en la mayor parte de las variables.

\section{Las diferencias según características} sociodemográficas de las madres

Ser una madre más mayor, estar en activo, y tener un nivel económico más bajo, son en la muestra del estudio variables que elevan el nivel de estrés de las madres. En la tabla que a continuación sigue se muestran la media, la desviación típica, y los valores mínimos y máximos del Índice de Estrés Parental, según los grupos de madres definidos por estas variables.

TABLA 1. DIFERENCIAS EN EL ESTRÉS DE LAS MADRES DE NIÑOS CON SÍNDROME DE DOWN SEGÚN PERFIL SOCIODEMOGRÁFICO

\begin{tabular}{|c|c|c|c|c|c|}
\hline & $\mathrm{N}$ & Mínimo & Máximo & Media & $\begin{array}{c}\text { Desviación } \\
\text { típica }\end{array}$ \\
\hline \multicolumn{6}{|l|}{ EDAD MADRE } \\
\hline $26-30$ & 5 & 53 & 78 & 63,00 & 9,97 \\
\hline $31-35$ & 23 & 39 & 108 & 63,73 & 18,97 \\
\hline $36-40$ & 54 & 36 & 106 & 61,64 & 15,85 \\
\hline $41-45$ & 43 & 43 & 151 & 75,41 & 26,53 \\
\hline $46-50$ & 27 & 39 & 126 & 71,59 & 18,92 \\
\hline $51-55$ & 9 & 44 & 124 & 73,55 & 26,61 \\
\hline \multicolumn{6}{|l|}{ SITUACIÓN LABORAL } \\
\hline En activo & 90 & 36 & 151 & 68,07 & 22,91 \\
\hline Desempleada & 56 & 39 & 126 & 68,94 & 19,49 \\
\hline En excedencia & 5 & 54 & 79 & 66,80 & 8,96 \\
\hline De baja por enfermedad & 1 & 48 & 48 & 48,00 & - \\
\hline Ama de casa & 9 & 41 & 106 & 64,22 & 20,63 \\
\hline
\end{tabular}


Periódico do Núcleo de Estudos e Pesquisas sobre Gênero e Direito Centro de Ciências Jurídicas - Universidade Federal da Paraíba Edição Especial - Health, Gender and Human Rights V. 6 - No 01 - Ano 2018

ISSN | 2179-7137 | http://periodicos.ufpb.br/ojs2/index.php/ged/index

\begin{tabular}{|l|c|c|c|c|c|}
\hline \multicolumn{2}{|l|}{ NIVEL DE INGRESOS } & 39 & 74,41 & 21,55 \\
\hline $\begin{array}{l}\text { Bajos (Hasta 1200 euros } \\
\text { mensuales) }\end{array}$ & 55 & 39 & 131 & \\
\hline $\begin{array}{l}\text { Medios (Entre 1200 y } \\
2400 \text { euros mensuales) }\end{array}$ & 45 & 40 & 151 & 66,11 & 21,70 \\
\hline $\begin{array}{l}\text { Altos (Más de 2400 } \\
\text { euros mensuales) }\end{array}$ & 61 & 36 & 144 & 63,60 & 19,36 \\
\hline
\end{tabular}

Fuente: Elaboración propia. Cuestionario de adaptación familiar con hijos con síndrome de Down entre 0 y 12 años (2014).

Es en los tres grupos de edad más jóvenes de nuestro estudio dónde la puntuación media de estrés es más baja, experimentado una elevación de entre 10 y 12 puntos a partir del intervalo de edad de 41 a 45 años. Son las madres de entre 26 y 30 años las que experimentan menos estrés. Aunque presentan una puntuación media ligeramente por encima del grupo de 36-40, ésta sigue siendo baja y la desviación típica es significativamente inferior al resto de grupos de edad. Todas ellas tienen puntuaciones en el Índice de Estrés entre 53 y 78 puntos. No obstante, el tamaño de este grupo de madres es muy pequeño.

Por el contrario, las mujeres entre 41 y 45 años tienen la puntuación media en la escala de estrés más alta, 75,41 puntos, aunque la dispersión que presentan es significativa. Tal y como se puede ver en los valores mínimo y máximo de la tabla, hay madres de esas edades con puntuaciones bajas de estrés y otras por el contrario con puntuaciones bastante altas.

Las madres del estudio desempleadas y en activo son las que presentan puntuaciones medias en estrés más altas, 68,94 y 68,07 puntos respectivamente, siendo no obstante su dispersión alta. El resto de grupos de madres tienen medias más bajas.

El nivel de ingresos es la característica que en nuestra muestra origina diferencias más claras en el nivel de estrés de las madres. A medida que aumenta el nivel de ingresos descienden las puntuaciones medias en estrés, teniendo desviaciones típicas similares. Las madres con un nivel de ingresos bajo presentan la media en el Índice de Estrés más alta - 74,41 puntos-, seguidas de las madres con ingresos medios - 66,11 puntos-, siendo aquellas con ingresos más altos las que tienen menos estrés 63,60 puntos-. 
Periódico do Núcleo de Estudos e Pesquisas sobre Gênero e Direito Centro de Ciências Jurídicas - Universidade Federal da Paraíba Edição Especial - Health, Gender and Human Rights V. 6 - $\mathrm{N}^{\mathrm{o}} 01$ - Ano 2018

ISSN | 2179-7137 | http://periodicos.ufpb.br/ojs2/index.php/ged/index

Las diferencias según la composición familiar

Las madres del estudio que afrontan la crianza de un hijo con síndrome de Down sin el otro progenitor son las que sufren de mayor estrés. La puntuación media en estrés es la más alta de los cuatro tipos de familia identificados $-78,52$ puntos -, superando a las madres que viven en familias biparentales en 12 puntos. Las madres que parecen tener menos estrés son las que viven en pareja y con otros familiares, dado que son las que tienen la media más baja y la desviación típica también más baja. Es decir, son las madres que son más iguales en unas puntuaciones bajas de estrés.

TABLA 2. DIFERENCIAS EN EL ESTRÉS DE LAS MADRES DE NIÑOS CON SÍNDROME DE DOWN SEGÚN COMPOSICIÓN FAMILIAR

\begin{tabular}{|c|c|c|c|c|c|}
\hline & $\mathrm{N}$ & Mínimo & Máximo & Media & $\begin{array}{c}\text { Desviación } \\
\text { típica }\end{array}$ \\
\hline \multicolumn{6}{|c|}{ TIPO FAMILIA } \\
\hline Biparental & 130 & 36 & 151 & 66,54 & 20,98 \\
\hline Monoparental & 23 & 52 & 131 & 78,52 & 21,76 \\
\hline Extensa & 4 & 56 & 79 & 67,50 & 11,21 \\
\hline Otra & 4 & 40 & 79 & 55,25 & 16,74 \\
\hline \multicolumn{6}{|c|}{ NÚMERO DE HIJOS } \\
\hline 1 & 38 & 44 & 151 & 69,89 & 21,76 \\
\hline 2 & 78 & 36 & 144 & 67,89 & 21,05 \\
\hline 3 & 29 & 39 & 124 & 65,48 & 22,84 \\
\hline 4 & 8 & 43 & 112 & 70,00 & 23,31 \\
\hline 5 & 8 & 46 & 89 & 65,00 & 16,14 \\
\hline 6 & 1 & 78 & 78 & 78,00 & - \\
\hline 7 & 1 & 69 & 69 & 69,00 & - \\
\hline \multicolumn{6}{|c|}{ EDAD DE LOS HIJOS CON SÍNDROME DE DOWN } \\
\hline $0-3$ & 59 & 36 & 151 & 60,71 & 21,70 \\
\hline $4-6$ & 46 & 46 & 131 & 73,54 & 19,66 \\
\hline $7-9$ & 24 & 43 & 126 & 69,50 & 19,77 \\
\hline $10-12$ & 32 & 40 & 124 & 72,34 & 20,47 \\
\hline
\end{tabular}

Fuente: Elaboración propia. Cuestionario de adaptación familiar con hijos con síndrome de Down entre 0 y 12 años (2014). 
En las madres del estudio el

número de hijos no define una pauta regular en relación con las diferencias en el estrés maternal. Tal y como se puede ver en la tabla anterior, aunque las diferencias son pequeñas, la puntuación media de las madres que tienen un solo hijo o hija -éste con síndrome de Down, es muy similar a las madres con 4 hijos, y a las madres con 7 hijos, y más alta que la de las madres con 2, 3 y 5 hijos. Son las madres con 6 hijos las que tienen la media más alta en el Índice de Estrés parental, 78 puntos.

Las madres de los niños más pequeños, de 0 a 3 años, son las que en nuestra muestra tienen menos estrés. $\mathrm{Su}$ puntuación media es de 60,71 puntos, entre 10 y 12 puntos por debajo del resto de grupos de madres. Los cuatro años del niño o niña parecen definir un umbral a partir del cual el estrés que las madres experimentan con la crianza de su hijo/a crece. De hecho, es el grupo de edad que tiene la media más alta $-73,54$ puntos-, para luego descender ligeramente cuando los hijos tienen entre 7 y 9 años, y volver a repuntar en el grupo de 10 a 12 años.

\section{El apoyo social $y$ el estrés de las madres de niños con síndrome de Down}

En la siguiente tabla se resumen los datos del Índice de Estrés parental de las madres del estudio según si tiene o no las siguientes fuentes de apoyo: familiares (padres, hermanos, cuñados...), amigos íntimos, compañeros de trabajo, grupos formales o informales en el vecindario, creencias religiosas, parroquia o comunidad religiosa, profesionales, grupos de apoyo y medios de comunicación. No se refleja si tienen hijos obviamente - todas son madres-, y tampoco si tienen pareja, dado que entendemos que lo fundamental es este sentido es criar a sus hijos con una pareja, y esta dimensión ya ha sido analizada al describir las diferencias en estrés de las madres biparentales y monoparentales. Algunas de las evidencias que proporciona este análisis descriptivo deberían ser consideradas con bastante prudencia, dado el reducido tamaño de algunos grupos de madres. 
Periódico do Núcleo de Estudos e Pesquisas sobre Gênero e Direito Centro de Ciências Jurídicas - Universidade Federal da Paraíba Edição Especial - Health, Gender and Human Rights V. 6 - $\mathrm{N}^{\mathrm{o}} 01$ - Ano 2018

ISSN | 2179-7137 | http://periodicos.ufpb.br/ojs2/index.php/ged/index

\begin{tabular}{l} 
TABLA 3. DIFERENCIAS EN EL ESTRÉS DE LAS MADRES DE NIÑOS CON SíNDROME DE DOWN SEGÚN \\
DISPOSICIÓN DE REDES DE APOYO \\
\hline \\
\hline
\end{tabular}

PARIENTES COMO PADRES, HERMANOS, CUÑADOS...

\begin{tabular}{|l|c|c|c|c|c|}
\hline Sí & 147 & 36 & 151 & 66,63 & 21,16 \\
\hline No & 14 & 67 & 124 & 82,28 & 16,09 \\
\hline AMIGOS ÍNTIMOS & 136 & 36 & 151 & 65,71 & 19,65 \\
\hline Sí & 25 & 44 & 131 & 80,44 & 25,13 \\
\hline No & 98 & 36 & 151 & 65,78 & 20,92 \\
\hline COMPAÑEROS DE TRABAJO & 63 & 41 & 131 & 71,44 & 21,32 \\
\hline Sí & \multicolumn{5}{|l|}{}
\end{tabular}

IMPLICACIÓN EN ALGÚN GRUPO FORMAL O INFORMAL EN VECINDARIO O COMUNIDAD

\begin{tabular}{|l|c|c|c|c|c|}
\hline Sí & 39 & 36 & 101 & 63,87 & 18,41 \\
\hline No & 122 & 39 & 151 & 69,31 & 21,92 \\
\hline
\end{tabular}

CREENCIAS RELIGIOSAS

\begin{tabular}{|l|c|c|c|c|c|}
\hline Sí & 126 & 36 & 124 & 67,63 & 18,48 \\
\hline No & 35 & 40 & 151 & 69,31 & 29,26 \\
\hline \multicolumn{7}{|l|}{ PERTENENCIA A UNA PARROQUIA O COMUNIDAD RELIGIOSA } \\
\hline Sí & 51 & 39 & 101 & 65,43 & 15,17 \\
\hline No & 110 & 36 & 151 & 69,19 & 23,44 \\
\hline CONTACTO CON PROFESIONALES & 156 & 36 & 151 & 67,41 & 20,66 \\
\hline Sí & 5 & 44 & 126 & 86,20 & 31,73 \\
\hline No &
\end{tabular}

PERTENENCIA A ALGÚN GRUPO ESPECIAL DE AYUDA

\begin{tabular}{|l|l|l|l|l|l|}
\hline Sí & 94 & 39 & 144 & 65,87 & 18,99 \\
\hline No & 67 & 36 & 151 & 70,98 & 23,77 \\
\hline
\end{tabular}

USO DE MEDIOS DE INFORMACIÓN Y COMUNICACIÓN

\begin{tabular}{|l|c|c|c|c|c|}
\hline Sí & 157 & 36 & 151 & 67,68 & 21,36 \\
\hline No & 4 & 76 & 83 & 80,50 & 3,10 \\
\hline
\end{tabular}

Fuente: Elaboración propia. Cuestionario de adaptación familiar con hijos con síndrome de Down entre 0 y 12 años (2014).

Son la existencia de apoyo social informal próximo y el apoyo de los profesionales los factores protectores más relevantes paras las madres de 
nuestro estudio. De hecho, las madres que tienen la media más alta en estrés comparando la disposición de apoyos de diferente tipo, son las que declaran no tener contacto con profesionales, 86,20 puntos. Su media supera en casi 19 puntos a la de las madres que sí lo tienen, 67,41 puntos. Por su parte, la puntuación media de aquellas madres que declaran no tener familiares como padres, hermanos, u otros próximos supera casi 16 puntos $-82,28$ puntos -, a la del grupo de madres que sí los tienen - 66,39 puntos. La misma situación se produce cuando se les pregunta por si tienen amigos íntimos: la media de las madres que no los tienen es de 80,44 frente a una media de 65,71 puntos en las madres que sí disponen de ellos (una diferencia de casi 15 puntos).

Las diferencias entre las medias son menores en el caso de la existencia de compañeros de trabajo - 5,66 puntos, o de grupos formales o informales en su vecindario o comunidad - 5,44 puntos-, pertenencia a algún grupo de ayuda 5,11 puntos-, o pertenencia a una parroquia o comunidad religiosa $-3,76$ puntos-, siendo no obstante la media de estrés mayor en las madres que no tienen estos apoyos. Las creencias religiosas no parecen, en nuestra muestra, tener un peso significativo para el estrés de las madres - 69,31 puntos las que dicen no tener creencias religiosas frente a los 67,63 de las madres que dicen que sí.

El uso de medios de información y comunicación televisión, radio, libros, revistas...como materiales de apoyo, sí parece ser importante como factor modulador del estrés. La media de las madres que los utilizan es de 67,68 puntos frente a los 80,50 de las que no lo hacen.

\section{Conclusiones.}

La llegada de un hijo o hija con síndrome de Down a las familias estudiadas les provoca un impacto. Sin embargo, aunque la literatura informa del mayor riesgo de estrés para los progenitores cuando su hijo/a presenta una discapacidad intelectual (BackerEriczen et al, 2005; Gupta y Kaur, 2010; Hasall et al. 2005, Mak y ho, 2007; Webster et al., 2008), éste no se refleja en los resultados obtenidos por las madres estudiadas.

Bien es cierto, que diferentes estudios han puesto de manifiesto que el estrés no afecta de igual manera a todas las familias con hijos/as con discapacidad intelectual, y que aquellas 
que tienen menores con síndrome de Down suelen presentar un menor riesgo de padecer estrés y mayor satisfacción familiar (Scott et al, 1997), a lo que se ha denominado la ventaja del síndrome de Down. Este aspecto es confirmado por los resultados de nuestro estudio.

Uno de los momentos que genera tensión y estrés es la comunicación del diagnóstico de síndrome de Down del hijo o hija recién nacido o por nacer. Diferentes estudios han señalado que para muchas familias éste es un momento traumático por el contenido de la noticia y por la forma en que se produce su comunicación (Green, 2002, Skotko, 2005, Torres y Maia, 2009). Las madres estudiadas tienen en su mayoría una buena percepción de la persona que le informó del diagnóstico y la información y orientación que se le ofreció. Sin embargo, casi un tercio de las madres estudiadas pone de manifiesto que la atención y la información que obtuvieron no fue satisfactoria, lo que sin duda habrá influido en la percepción de la llegada de su hijo o hija con síndrome de Down como una situación estresante o cuanto menos, llena de tensión.

Las madres estudiadas disponen de recursos personales y acceso a recursos de apoyo social formal $\mathrm{e}$ informal para hacer frente a la crianza de su hijo o hija con síndrome de Down,. La mayor parte de las madres estudiadas comparten las tareas de crianza con una pareja con la que conviven, conformando familias biparentales. Sin embargo, algunos estudios han señalado las mayores posibilidades que tiene un menor con discapacidad intelectual de ser criado en un hogar monoparental (Loft, 2011, 2013; Manjarrés, 2012), por la ruptura de pareja (Hogan, et al., 2012) que puede derivarse de las dificultades de adaptación producidas a raíz de su nacimiento. Poseen un nivel educativo medio-alto, lo que se acompaña igualmente de niveles de renta familiar medios-altos mediante su participación en el empleo.

Asimismo las madres
estudiadas cuentan con apoyos
informales en el entorno familiar, disponen de amigos íntimos y están apoyados por profesionales. Las madres muestran una gran satisfacción por la atención que les dispensaron los profesionales de las entidades que trabajan con personas con discapacidad, tanto en su acogida como en las informaciones $y$ orientaciones dispensadas, en la línea de lo manifestado por Skotko y Bedia (2004). 
En este sentido, las evidencias de nuestra muestra apoyan que la percepción positiva de la acción de los profesionales funciona como una variable moduladora importante sobre la angustia y el choque que experimentan las familias al recibir la noticia. Dicha tensión, angustia y estrés pueden ser aumentados o aliviados con el buen apoyo y acompañamiento familiar por parte de los profesionales que, sin duda, es un recurso de protección en el proceso de adaptación familiar.

Las situaciones de mayor riesgo en el estrés maternal se producen cuando la madre es de más edad, está en el mercado de trabajo -ya sea ocupada o desempleada-, lo que le lleva a asumir una mayor carga, puesto que se ocupan dela mayor parte de las tareas de cuidado y crianza del menor con discapacidad (Núñez, 2010). Asimismo, presentan mayor riesgo de estrés las familias que disponen de pocos recursos económicos, afrontan la crianza las mujeres solas, y no cuentan con familiares y amigos próximos, ni tampoco con apoyo de profesionales.

En nuestra muestra la presencia de hermanos parece ejercer un papel protector para el estrés de las madres, que tiende a desaparecer a medida que aumenta el número de hijos. Sin embargo, este hallazgo ha de ser tomado con prudencia debido al escaso tamaño de los grupos de madres con más hijos, y a que la relación entre las variables estrés y número de hijos no es estrictamente lineal. Del mismo modo, madres cuyos hijos/as con síndrome de Down son más pequeños tienen menos estrés.

Finalmente, es necesario destacar que las mujeres estudiadas presentan niveles bajos de estrés, que se relacionan con la ventaja del síndrome de Down, y con la presencia de factores protectores frente al estrés parental como son el elevado nivel educativo y económico, la pertenencia a una familia biparental y la presencia de importantes recursos personales, tanto formales como informales. Aun así, es importante tener en cuenta a ese grupo de madres, aunque minoritario en nuestra investigación, que encabeza familias monoparentales con bajos recursos económicos y con pocos recursos informales para poder afrontar de manera satisfactoria la crianza de su hijo o hija con síndrome de Down. Urge que estas madres sean objeto de atención y especial protección por las distintas 
administraciones públicas y entidades

privadas del sector.

\section{Referencias Bibliográficas}

Abery, B. H. (2006). Family adjustment and adaptation with children with Down syndrome. Focus on Exceptional Children, 38 (5): 1-19.

Abidin, R. R. (1983). Parenting Stress Index Manual. Charlottesville, V. A.: Pediatric Psychology Press.

Abidin, R. R. (1992.) The determinants of parenting behaviour. Journal of Clinical Child Psychology, 21 (4): 407-412.

Abidin, R. R. (1995). Parenting Stres Index (PSI) manual. Charlottesville, VA: Pediatric Psychology Press.

Acero Rodríguez, P. D. (2013). Discapacidad: cómo afrontar el duelo en la familia. Madrid: San Pablo.

Aldosari, M. S. y Pufpaff, L. A. (2014). Sources of stress among parents of children with intellectual disabilities: a preliminary investigation in Saudi Arabia. The Journal of special eduction Apprenticeship, 3 (1): 1-21.

Antonovsky, A. (1987). Unraveling the Miystery of Health: How People Manage
Stress and Stay Well. San Francisco, CA.: Jossey-Bass Publishers.

Azar, M., y Badr, L. K. (2010). Predictors of coping in parents of children with an intellectual disability: Comparison between lebanese mothers and fathers. Journal of Pediatric Nursing, 25 (1): 46-56.

Azar, M. y Kurdahi, L. (2006). The adaptation of mothers of children with intellectual disability in Lebanon. Journal of Transcultural Nursing, 17 (4):375-380.

Baker-Ericzén, M. J. et al. (2005) Stress levels and adaptability in parents of toddlers with and without autism spectrum disorders. Research and practice for prsons with severe disabilities, 30 (4): 194-204.

Basa, J. A. (2010) Estrés parental con hijos autistas. Un estudio comparativo. Tesis de Licenciatura, Universidad Católica Argentina, Facultad de Humanidades "Teresa de Ávila", Argentina. Recuperado en marzo de 2014: http://bibliotecadigital.uca.edu.ar/repositori o/tesis/estres-parental-con-hijosautistas.pdf.

Blacher, J. et al. (2013). Syndrome specificity and mother-child interactions: Examining positive and negative parenting across contexts and time. Journal of Autism 
Periódico do Núcleo de Estudos e Pesquisas sobre Gênero e Direito

Centro de Ciências Jurídicas - Universidade Federal da Paraíba

Edição Especial - Health, Gender and Human Rights

V. 6 - No 01 - Ano 2018

ISSN | 2179-7137 | http://periodicos.ufpb.br/ojs2/index.php/ged/index

and Developmental Disorders, 43: 761-774.

Bower, A., Chant, D., \& Chatwin, S. (1998).

Hardiness in families with and without a child with Down syndrome. Down Syndrome Research and Practice, 5 (2): 7177.

Byrne, E. A. \& Cunningham, C. C. (1985). The effects of mentally handicapped children on families: A conceptual review. Journal of Child Psychology and Psychiatry and Allied Disciplines, 26 (6): 847- 864.

Canbulat, N., et al. (2014). Emotional reactions of mothers diagnosed with Down syndrome baby. International Journal of Nursing Knowledge, 25 (3): 147-153.

Cea D’Ancona, M. A. (1999). Metodología cuantitativa. Estrategias y técnicas de investigación social. Madrid: Síntesis.

Chichevska Jovanova, N. y Dimitrova Radojichikj, D. (2013) Parents of children with developmental disabilities: stress and support. Special Education-Profesional and Scientific Issues, 14 (1-2): 7-19.

Choi, E.K., et al.. (2011). Factors associated with emotional response of parents at the time of diagnosis of Down syndrome. Journal for Specialists in Pediatric Nursing, 16: 113-120.
Cooke, K., et al. (1986). Child disablement, family dissolution and reconstitution. Developmental Medicine and Child Neurology, 28: 610-16.

Corbetta, P. (2007). Metodología y técnicas de investigación social. Madrid: McGraw Hill.

Cunningham, C. C. (1996). Families of children with Down syndrome. Down Syndrome Research and Practice, 4 (3): 87 95.

Cunningham, C. (2011). El síndrome de Down. Una introducción para padres. Barcelona: Paidós.

Dabrowska, A. y Pisula, E. (2010). Parenting stress and coping styles in mothers and fathers of pre-school children with autism and Down syndrome. Journal of Intellectual Disability Research, 54(3): 266-280.

Di Giulio, P. et al. (2014). Families with disabled children in different european countries. Families and societies, 23. Recuperado en abril de 2015: http://www.familiesandsocieties.eu/wpcontent/uploads/2014/12/WP23GiulioEtAl. pdf

Díaz-Herrero, A., et al. (2010). Estructura factorial y consistencia interna de la versión española del Parenting Stress Index-Short Form. Psicothema, 22 (4): 1033-1038. 
ISSN | 2179-7137 | http://periodicos.ufpb.br/ojs2/index.php/ged/index

Díaz-Herrero, A., et al. (2011). Validity of the Parenting Stress Index-Short Form in a sample of spanish fathers. The Spanish Journal of Psychology, 14 (2): 990-997.

Dubbs, J. L. (2008). Parent stress reduction through a psychosocial intervention for children diagnosed with attentiondeficit/hiperactivity disoreder. A Dissertation Submitted to the School of Graduate Studies and Research In Partial Fulfillment of the Reuirements for the Degree Doctor of Psychology. Indiana University of Pennsylvania. Recuperado en abril de 2014https://dspace.iup.edu/bitstream/handle /2069/75/Jenna\%20Dubbs.pdf?sequence=1

Dyson, L .L. (1991). Families of young children with handicaps: Parental stress and family functioning. American Journal on Mental Retardation, 95: 623-629.

Dyson, L. L. (1997) Fathers and mothers of school-age children with developmental disabilities: parental stress, family functioning, and social support. American Journal on Mental Retardation, 102: 267279.

Emerson, E. (2003). Mothers of children and adolescents with intellectual disability: social and economic situation, mental health status, and the self-assessed social and psychological impact of the child's difficulties. Journal of Intellectual

Disability Research, 47: 385-399.

FEAPS (2008). Necesidades de las Familias de Personas con Discapacidad Intelectual de Feaps Madrid. Madrid: FEAPS.

Flórez, J. (2000). A la vera de nuestros caminos. Evocaciones sobre la discapacidad. Santander: Fundación Síndrome de Down Cantabria.

Friedrich, W. N. y Friedrich, W. L. (1981). Psychosocial assets of parents of handicapped and nonhandicapped children. American Journal of Mental Deficiency, 85(5): 551-553.

GAT (2011). La Primera Noticia. Madrid, Polibea.

Gath, A. (1978). Down's Syndrome and the family: he early years. London: Academic Press.

Glidden, L. M., y Floyd, F. J. (1997). Disaggregatin parental depression and family stressin assessing families of children with developmental disabilities: a multisample aalysis. American Journal on Mental Retardation, 102: 250-266.

Gray, D. E. (2003). Gender and coping: The parents of children with high functioning autism. Social Science and Medicine, 56: 631-642. 
Periódico do Núcleo de Estudos e Pesquisas sobre Gênero e Direito Centro de Ciências Jurídicas - Universidade Federal da Paraíba Edição Especial - Health, Gender and Human Rights V. 6 - No 01 - Ano 2018

ISSN | 2179-7137 | http://periodicos.ufpb.br/ojs2/index.php/ged/index

Green, S. E. (2002). Mothering Amanda:

Musing on the experience of raising a child with cerebral palsy. Journal of Loss and Trauma, 7: 21-34.

Gupta, R. K. y Kaur, H. (2010). Stress among parents of children with intellectual disability. Asia Pacific Disability Rehabilitation Journal, 21(2): 118-126.

Hassall, R., Rose, J., y McDonald, J. (2005). Parenting stress in mothers of children with an intellectual disability: the effects of parental cognitions in relation to child characteristics and family support. Journal of Intellectual Disability Research, 49 (6): 405-418.

Hastings, R. (2002). Parental stress and behavior problems of children with developmental disabilities. Journal of Intellectual \& Developmental disability, 2: 149-160.

Hastings, R. P., et al.. (2002). Factors related to positive perceptions in parents of children with intellectual disabilities. Journal of Applied Research in Intellectual Disabilities, 15 (3), 269-275.

Hastings, R. P., et al. (2006). Maternal distress and expressed emotion: crosssectional and longitudinal relationships with behavior problems of children with intelectual disabilities. American Journal on
Mental Retardation, 11: 48-61.

Hastings, R. P. et al. (2005). Coping strategies in mothers and fathers of preschool and school-age children with autism. Autism, 9 (4): 377-391.

Hedov, G., et al.. (2000). Self-perceived health in Swedish parents of children with Down's syndrome. Quality of Life Research, 9 (4): 415-422.

Hedov, G.; et al. (2002) First information and support provided to parents of children with Down syndrome in Sweden: clinical goals and parental experiences. Acta Paediatr, 91: 1344-1349.

Hodapp, R. M. (2007). Families of persons with Down syndrome: new perspectives, findings, and research and service needs. Mental Retardation and Developmental Disabilities Research Reviews 13: 279 287.

Hodapp, R. M., et al. (2003). The effects of the child with Down syndrome on maternal stress. British Journal of Developmental Psychology, 22: 137-151.

Hogan, D., et al. (2012). Family consequences of children's disabilities. Russell: Sage Foundation.

Johnston, C., et al. (2003). Factors 
ISSN | 2179-7137 | http://periodicos.ufpb.br/ojs2/index.php/ged/index

associated with parentign stress in mothers of children with fragile X syndrome. Journal of Developmental and Behavioral Pediatrics, 24 (4): 267-275.

Keller, D., y Honig, A. S. (2004). Maternal and paternal stress in families with schoolaged children with disabilities. American Journal of Orthopsychiatry, 74 (3): 337-348.

Kearney, P. M., y Griffin, T. (2001).

Between joy and sorrow: Being a parent of a child with developmental disability. Journal of Advanced Nursing, 34: 582592.

Kersh, J. et al. (2006). The contribution of marital quality to the wellbeing of parents of children with developmental disabilities. Journal of Intellectual Disability Research, 50 (12): 883-893.

Lalvani, P. (2008). Mothers of children with Down syndrome: Constructing the sociocultural meaning of disability. Intellectual and Developmental Disabilities, 46 (6), 436-445.

Lalvani, P. (2011). Constructing the (m)other. Dominant and contested narratives on mothering a child with Down syndrome. Narrative Inquiry, 21 (2): 276-293.

Loft, L. T. G. (2013). Four Essays on Family
Life Events. Recuperado en mayo de 2014: http://www.soc.ku.dk/Forskning/dokument/ thesis/FraForlag06_12_13.pdf.

Lott, I. T. y Dierssen, M. (2010). Cognitive deficits and associated neurological complications in individual with Down's syndrome. Lancet Neurology, 9: 623-633.

Mak, W. S. y Ho, S. M. (2007) Caregiving perceptions of Chinese mothers of children with intellecutal disability in Hong Kong. Journal of Applied Research in Intellecutal Disabilities, 20: 145-456.

Manjarrés Carrizales, D. (2012). Apoyo y fortalecimiento a familias para la crianza de niños con discapacidad. Horizontes Pedagógicos, 14 (1): 97-118.

Martínez-Pampliega, A., et al. (2006). Family Adaptability and Cohesion Evaluation Scale (FACES): desarrollo de una versión de 20 items en español. International Journal of Clinical and Health Psychology, 6 (2): 317-338

McCubbin, H. I. y Patterson, J. M. (1983). The family stress process: the double ABCX model of adjustment and adaptation. Marriage and Family Review, 6: 7-37

McCubbin, M. A., y McCubbin, H. I. (1993). Families coping with illness: The resiliency model of family stress, adjustment and 
Periódico do Núcleo de Estudos e Pesquisas sobre Gênero e Direito Centro de Ciências Jurídicas - Universidade Federal da Paraíba Edição Especial - Health, Gender and Human Rights V. 6 - No 01 - Ano 2018

ISSN | 2179-7137 | http://periodicos.ufpb.br/ojs2/index.php/ged/index

adaptation. En Danielson, C. B., HamelBissell, B. \& Winstead-Fry, P. Families, Health and Illness (pp. 21-63) St. Louis, MO: CV Mosby.

Mercado García, E. y García Vicente, L. M. (2010). Necesidades sociales de las personas con discapacidad en edad escolar y sus familias. Cuadernos de Trabajo Social, 23: $9-24$.

Minnes P. M. (1988) Family resources and stress associated with having a mentally retarded child. American Journal on Mental Retardation 93: 184- 92.

Minnes, P. (1998). Mental retardation: the impact upon the family. En Burack, J. A., Hodapp, R. M., \& Zigler, E. (Coord.), Handbook of mental retardation and development (pp. 693-712). Cambridge: Cambridge University Press.

Neely-Barnes, S. L. y Dia, D. A. (2008). Families of children with disabilities: a review of literature and recommendations for interventions. Journal of Early and Intensive Behavior intervencion, 5 (3): 93107.

Negrin, N. S. y Cristante, F. (1996). Resources and stress in parents with a mentally retarded child: a quantitative approach. En Cusinato, M. (Coord.). Research on family: resources and needs across the world (pp. 493-506). Milão: LED

- Edicioni Universitarie.

Núñez, B. (2010). El niño con discapacidad, la familia y su docente. Buenos Aires: Lugar.

Oelofsen, N. y Phil Richardson, P. (2006) Sense of coherence and parenting stress in mothers and fathers of preschool children with developmental disability. Journal of Intellectual and Developmental Disability, 31 (1): 1-12.

Olsson, M. B. y Hwang, C. P. (2001). Depression in mothers and fathers of children with intellectual disability. Journal of intellectual Disability Research, 45 (6): 535- 543.

Ortega, P., Salguero, A., y Garrido Garduño, A. (2007). Discapacidad: paternidad y cambios familiars. Avances en Psicología Latinoamericana/Bogotá (Colombia), 25 (1): 118-125.

Padeliadu, S. (1998). Time demands and experience stress in greek mothers of children with Down's syndrome. Journal of Intellectualy Disability, 42 (2): 144-153.

Phelps, R. A. et al. (2012) Health care needs of children with Down syndrome and impact of health system performance on children and their families. Journal of Developmental and Behavioral Pediatrics, 33: 214-220. 
Periódico do Núcleo de Estudos e Pesquisas sobre Gênero e Direito Centro de Ciências Jurídicas - Universidade Federal da Paraíba Edição Especial - Health, Gender and Human Rights V. 6 - No 01 - Ano 2018

ISSN | 2179-7137 | http://periodicos.ufpb.br/ojs2/index.php/ged/index

Pereira Silva, N. L. y Dessen, M. A. (2003). Cianças preescolares com sindrome de Down e suas interaçones familiares. Psicologia: Reflexao e critica, 16 (3): 503514.

Pereira Silva, N. L. (2006). Familias de crianças com síndrome de Down: sentimentos, modos de vida e estresse parental. Interaçao em Psicología, 10 (2): 183-194

Pozo Cabanillas, P., Sarriá Sánchez, E., \& Méndez Zaballos, L. (2006). Estrés en madres de personas con trastornos del espectro autista. Psicothema, 18 (3): 342347

Rasheed, J. M., Rasheed, M. N., y Marley, J. A. (2001). Family therapy. Models and tecniques. London: Sage.

Robledo, J. V. (2011). Construir un nuevo proyecto de vida familiar: la irrupción de un hijo con discapacidad y el impacto de su diagnóstico. Tesina. Universidad Nacional del Rosario.

Rodrigues Nunes, M. D., y Dupas, G. (2011). Independence of children with Down syndrome: the experiences of families. Revista Latino-Americana de Enfermagem, 19 (4): 985-993.
Rogers, M. L. y Hogan, D. P. (2003) Family life with children with disabilities: the key role of rehabilitation. Journal of Marriage and Family, 65: 818-833.

Ruano Piera, R. y Serra Desfilis, E. (2000). Estrategias de afrontamiento en familias con hijos adolescentes. Anales de Psicología, 16 (2): 199-206.

Sanders, J. L. y Morgan, S. B. (1997) Family stress and adjustment as perceived by parents of children with autism or Down Syndrome: Implications for Intervention. Child and Family Behavior Therapy, 19: 1532.

Sanz Vázquez, M. (2008). Equipo EIF. Manual de Instrumentos de evaluación familiar. (pp. 69- 77).Madrid: CCS.

Sayed, M. et al. (2006). Parenting Stress Index among Mothers of Conduct Disorder Children. Current Psychiatry, 13 (2): 255270.

Scorgie, K. y Sobsey, D. (2000). Transformational outcomes associated with parenting children who have disabilities. Mental Retardation, 38 (3): 195-206.

Scott, B. S. et al. (1997). Psychological distress of parents of infants with Down syndrome. American Journal on Mental Retardation, 102 (2): 161-171. 
Periódico do Núcleo de Estudos e Pesquisas sobre Gênero e Direito Centro de Ciências Jurídicas - Universidade Federal da Paraíba Edição Especial - Health, Gender and Human Rights V. 6 - No 01 - Ano 2018

ISSN | 2179-7137 | http://periodicos.ufpb.br/ojs2/index.php/ged/index

Shapiro, J. et al. (1998). Maternal reactions to children with mental retardation. En Burack, J. A., Hodapp R. M. \& Zigler E. (Coord.). Handbook of mental retardation and development (pp. 606-636). Cambridge: Cambridge University Press.

Shin, J. et al. (2006). Parenting Stress of mothers and fathers of young children with cognitive delays in Vietnam. Journal of Intellectual Disability Research, 50: 748760.

Shonkoff, J. P. et al. (1992). Development of infants with disabilities and their families: Implications for theory and service delivery. Monogrphs of the Society ofr Research in Child Development, 57 (6, Serial No. 230).

Silva, N. L. P. y Dessen, M. A. (2003). Ciranças com síndrome de Down e suas interaçoes familiares. Psicología: $R$ eflexao e Crítica, 16(3): 503-514.

Simmerman, S. et al. (2001). Fathers' and mothers' perceptions of father involvement in families with Young children with a disability. Journal of Intellectual and Developmental Disability, 26: 325-338.

Singer, G. (2006). Meta-analysis of comparative studies of depression in mothers of children with and without developmental disabilities. American
Journal on Mental Retardation, 111 (3): 155-169.

Skotko, B. G. (2005) Mothers of children with Down syndrome reflect on their postnatal support. Pediatrics, 115: 64-77.

Skotko, B. y Bedia, R. C. (2004). Apoyo postnatal para madres de niños con síndrome de Down. Revista Síndrome de Down, 21: 54-71.

Skotko, B. et al. (2005) Postnatal support for mothers of children with Down syndrome. Mental Retardation, 43 (3): 196-212.

Tomanik, S. et al. (2004). The relationship between behaviors exhibited by children with autism and maternal distress. Journal of Intellectual \& Developmental Disability, 29 (1): 16-26.

Torres, L. y Maia, C. (2009). Percepción de las madres acerca del contenido de la información del diagnostico de síndrome de Down. Revista Chilena de Pediatría, 80 (1): 39-47.

Webster, T. I. et al. (2008). Child health and parental stress in school-age children with a preschool diagnosis of developmental delay. Journal of Child Neurology, 23 (1): 32-38. 